VOL. 59 (1999) [237-246]

\title{
THE RANGE OF THE HELGASON-FOURIER TRANSFORMATION ON HOMOGENEOUS TREES
}

\author{
Michael Cowling and Alberto G. Setti
}

Let $\mathfrak{X}$ be a homogeneous tree, $o$ be a fixed reference point in $\mathfrak{X}$, and $\mathfrak{B}_{N}$ be the closed ball of radius $N$ in $\mathfrak{X}$ centred at $o$. In this paper we characterise the image under the Helgason-Fourier transformation $\mathcal{H}$ of $C_{N}(\mathfrak{X})$, the space of functions supported in $\mathfrak{B}_{N}$, and of $S(\mathfrak{X})$, the space of rapidly decreasing functions on $\mathfrak{X}$. In both cases our results are counterparts of known results for the Helgason-Fourier transformation on noncompact symmetric spaces.

Let $\mathfrak{X}$ be a homogeneous tree of degree $q+1$, that is, a connected graph with no loops in which every vertex is adjacent to $q+1$ other vertices. We denote by $o$ a fixed reference point in $\mathfrak{X}$, by $|x|$ the distance of $x$ from $o$, that is, the number of edges between $o$ and $x$, by $G$ the automorphism group of $\mathfrak{X}$, and by $K$ the stabiliser of $o$ in $G$. The boundary $\Omega$ of $\mathfrak{X}$ may be identified with the set of infinite geodesic rays issuing from $o$. We write $\mathfrak{B}_{N}$ and $\mathfrak{S}_{N}$ for the closed ball $\{x \in \mathfrak{X}:|x| \leq N\}$ and the sphere $\{x \in \mathfrak{X}:|x|=N\}$. By $\mathfrak{B}_{-1}$ we mean the empty subset of $\mathfrak{X}$.

If $x$ and $y$ are in $\mathfrak{X}$ and $\omega$ is in $\Omega$, we define $c(x, \omega)$ to be the confluence point of $x$ and $\omega$, that is, the last point lying on $\omega$ in the geodesic path $\left\{o, x_{1}, x_{2}, \ldots, x\right\}$ joining $o$ to $x$, and define similarly the confluence point $c(x, y)$. The height $h_{\omega}(x)$ of $x$ in $\mathfrak{X}$ with respect to $\omega$ is defined by the formula

$$
h_{\omega}(x)=2|c(x, \omega)|-|x| .
$$

Clearly, $h_{\omega}(x) \leq|x|$. On the boundary $\Omega$ there is a natural $K$-invariant, $G$-quasiinvariant probability measure $\nu$, and the Poisson kernel $p(g o, \omega)$ is defined to be the Radon-Nikodym derivative $d \nu\left(g^{-1} \omega\right) / d \nu(\omega)$. Then

$$
p(x, \omega)=q^{h_{\omega}(x)} \quad \forall x \in \mathfrak{X} \quad \forall \omega \in \Omega ;
$$

see, for example, [4, Chapter 2], or [3, Section 2]. We define $E_{i}(x)$ to be the set of $\left\{\omega^{\prime} \in \Omega:\left|c\left(x, \omega^{\prime}\right)\right|=i\right\}$; then $\nu\left(E_{i}(x)\right) \leq q^{-i}$, and

$$
p(x, \omega)=\sum_{j=0}^{|x|} q^{2 j-|x|} \chi_{E_{j}(x)}(\omega) \quad \forall x \in \mathfrak{X} \quad \forall \omega \in \Omega ;
$$

Received 14th July, 1998

Copyright Clearance Centre, Inc. Serial-fee code: 0004-9729/99 \$A2.00+0.00. 
see $[\mathbf{7},(2.3)]$ or $\left[\mathbf{5}\right.$, Proposition 2.5]. We write $E(x)$ for $E_{|x|}(x)$, and define the averaging operators $\mathcal{E}_{n}$ on $C(\Omega)$ by the formulae $\mathcal{E}_{-1}=0$ and, when $n \geq 0$,

$$
\mathcal{E}_{n} \eta(\omega)=\nu(E(x))^{-1} \int_{E(x)} \eta(\omega) d \nu(\omega) \quad \forall x \in \mathfrak{S}_{n} \quad \forall \omega \in E(x) .
$$

We define, for $z$ in $\mathbb{C}$, representations $\pi_{z}$ of $G$ on $C(\Omega)$ by the formula

$$
\left[\pi_{z}(g) \eta\right](\omega)=p^{1 / 2+i z}(g o, \omega) \eta\left(g^{-1} o\right) \quad \forall g \in G \quad \forall \omega \in \Omega .
$$

It is clear that $\pi_{z}=\pi_{z+\tau}$, where $\tau=2 \pi / \log q$. We write $\mathbb{T}$ for the torus $\mathbb{R} / \tau \mathbb{Z}$, which we usually identify with the interval $[-\tau / 2, \tau / 2)$. The Poisson transformation $\mathcal{P}^{z}$ : $C(\Omega) \rightarrow C(\mathfrak{X})$ is given by the formula

$$
\mathcal{P}^{z} \eta(x)=\left\langle\pi_{z}(x) 1, \eta\right\rangle=\int_{\Omega} p^{1 / 2+i z}(x, \omega) \eta(\omega) d \nu(\omega) .
$$

The spherical function $\phi_{z}$ on $\mathfrak{X}$ is defined to be $\mathcal{P}^{z} 1$. It is known that

$$
\phi_{z}(x)= \begin{cases}\left(\frac{q-1}{q+1}|x|+1\right) q^{-|x| / 2} & \forall z \in \tau \mathbb{Z} \\ \left(\frac{q-1}{q+1}|x|+1\right) q^{-|x| / 2}(-1)^{|x|} & \forall z \in \tau / 2+\tau \mathbb{Z} \\ \mathbf{c}(z) q^{(i z-1 / 2)|x|}+\mathbf{c}(-z) q^{(-i z-1 / 2)|x|} & \forall z \in \mathbb{C} \backslash(\tau / 2) \mathbb{Z},\end{cases}
$$

where $\mathbf{c}$ is the meromorphic function given by

$$
\mathbf{c}(z)=\frac{q^{1 / 2}}{q+1} \frac{q^{1 / 2+i z}-q^{-1 / 2-i z}}{q^{i z}-q^{-i z}} \quad \forall z \in \mathbb{C} \backslash(\tau / 2) \mathbb{Z} .
$$

Now

$$
\phi_{0}(x)=\int_{\Omega} p^{1 / 2}(x, \omega) d \nu(\omega)=\sum_{x \in \mathfrak{S}_{n}} \int_{E(x)} q^{h_{\omega}(x) / 2} d \nu(\omega)
$$

whence

$$
\sum_{x \in \mathfrak{S}_{n}} q^{h_{\omega}(x) / 2} \leq 2(n+1) q^{-n / 2} \quad \forall n \in \mathbb{N} .
$$

It should perhaps be remarked that we use a different parametrisation of the representations and spherical functions from Figà-Talamanca and his collaborators (for example, [5] and [4]): our $\phi_{z}$ corresponds to their $\phi_{1 / 2+i z}$, and $\pi_{z}$ and $\mathrm{c}(z)$ are similarly reparametrised. Similar comments apply to the intertwining operators considered below. Our parametrisation makes the analogy with the semisimple Lie group case more transparent. 
The Helgason-Fourier transform $\tilde{f}$ of a finitely supported function $f$ on $\mathfrak{X}$ is the function on $\mathbb{T} \times \Omega$ defined by the formula

$$
\tilde{f}(s, \omega)=\left[\pi_{s}(f) 1\right](\omega)=\sum_{x \in \mathcal{X}} f(x) p^{1 / 2+i s}(x, \omega)
$$

The Helgason-Fourier transformation $\mathcal{H}$ is the linear operator that maps $f$ to $\tilde{f}$. The following inversion and Plancherel formulae hold (see [5, Chapter 3 Section IV and Chapter 5 Section IV], or [4, Chapter II Section 6]). If $f$ is finitely supported on $\mathfrak{X}$, then

$$
f(x)=\int_{J_{\mathbb{T}}} \int_{J_{\Omega}} \underline{p}^{1 / 2-i s}(x . \omega) \tilde{f}(s . \omega) d \nu(\omega) d \mu(s) \quad \forall x \in \mathfrak{X}
$$

If $f_{1}$ and $f_{2}$ are finitely supported, then

$$
\sum_{x \in X} f_{1}(x) \overline{f_{2}}(x)=\int_{\mathbb{T}} \int_{\Omega} \tilde{f}_{1}(s, \omega) \overline{\widetilde{f}_{2}(s, \omega)} d \nu(\omega) d \mu(s)
$$

The Helgason-Fourier transformation extends to an isometric mapping from $L^{2}(\mathfrak{X})$ into $L^{2}(\mathbb{T} \times \Omega, \mu \times \nu)$, so $\mathcal{H}$ is injective on $L^{2}(\mathfrak{X})$. Its range is then the subspace of $L^{2}(\mathbb{T} \times \Omega, \mu \times \nu)$ of the functions $F$ which satisfy the symmetry condition

$$
\int_{\Omega} p^{1 / 2-i s}(x, \omega) F(s, \omega) d \nu(\omega)=\int_{\Omega} p^{1 / 2+i s}(x, \omega) F(-s, \omega) d \nu(\omega)
$$

for every $x$ in $\mathfrak{X}$ and almost every $s$ in $\mathbb{T}$. Here, $\mu$ denotes" the Plancherel measure, whose density with respect to Lebesgue measure is given by $c_{G}|\mathbf{c}(s)|^{-2}$ (see, for example, [5] or [4]). We note that $\mathbf{c}^{-1}$ is smooth on $\mathbb{T}$.

The space of functions supported in $\mathfrak{B}_{N}$ is written $C_{N}(\mathfrak{X})$. A function $f$ on $\mathfrak{X}$ is said to be rapidly decreasing if, for every $k$ in $\mathbb{N}$, there exists a constant $C_{k}$ such that

$$
|f(x)| \leq C_{k}(|x|+1)^{-k} q^{-|x| / 2} \quad \forall x \in \mathfrak{X}
$$

(see, for example, [1]). The space of rapidly decreasing functions is denoted by $S(\mathfrak{X})$.

The aim of this paper is to characterise the image under $\mathcal{H}$ of the spaces $C_{N}(\mathfrak{X})$ and $S(\mathfrak{X})$. After a preliminary version of this paper was completed, we learned that a similar characterisation of the range of $C_{N}(\mathfrak{X})$, involving the horocyclical Radon transformation $\mathcal{R}$ on $\mathfrak{X}$, was obtained independently by Tarabusi, Cohen, and Colonna [2]; these authors also describe the the image under $\mathcal{R}$ of certain spaces of "slowly vanishing functions" on $\mathfrak{X}$. We refer to [3, Section 2] for a discussion of the relationship between $\mathcal{R}$ and $\mathcal{H}$. 


\section{FunCtions With FINITE SUPPORT}

It is easy to see that, if $f$ is in $C_{N}(\mathfrak{X})$, then the following conditions hold:

(i) $\tilde{f}$ is continuous on $\mathbb{T} \times \Omega$ (indeed, $\tilde{f}$ is in $C^{\infty}(\mathbb{T} \times \Omega)$ in the sense of Theorem 2 below);

(ii) $\tilde{f}$ extends to a $\tau$-periodic entire function of exponential type $N$ uniformly in $\omega$, that is, there exists $C$ such that

$$
|\widetilde{f}(z, \omega)| \leq C q^{|\operatorname{Im} z| N} \quad \forall \omega \in \Omega \quad \forall z \in \mathbb{C} ;
$$

(iii) $\tilde{f}$ satisfies the symmetry condition (2);

(iv) $\tilde{f}$ is $N$-cylindrical in $\omega$, that is, for $s$ fixed, $\tilde{f}(s, \omega)$ is constant on the sets $E(x)$ for every $x$ in $\mathfrak{S}_{N}$.

Conditions (i)-(iii) are the analogues of the conditions that describe the Paley-Wiener space for the Helgason-Fourier transformation (see [6]). The content of the following theorem is that (i)-(iii) characterise the image of $C_{N}(\mathfrak{X})$ under $\mathcal{H}$.

TheOREM 1. A function $F: \mathbb{T} \times \Omega \rightarrow \mathbb{C}$ is the Helgason-Fourier transform of a function $f$ in $\mathbb{C}_{N}(\mathfrak{X})$ if and only if $F$ satisfies conditions (i)-(iii).

Proof: Clearly only the "if" implication requires proof. It should be noted that, contrary to the symmetric space case and to the case of radial functions on $\mathfrak{X}$, the proof is not obtained by contour integration arguments alone, but also involves a counting argument.

Since $\mathcal{H}$ is injective, $\mathcal{H}\left(C_{N}(\mathfrak{X})\right)$ has dimension equal to the cardinality $\left|\mathfrak{B}_{N}\right|$ of $\mathfrak{B}_{N}$, and it suffices to show that the space of functions on $\mathbb{T} \times \Omega$ which satisfy conditions (i)-(iii) has dimension at most (and therefore exactly) $\left|\mathfrak{B}_{N}\right|$.

To do this, we recast the symmetry condition (2) in a more suitable form. Using the representations $\pi_{z}$ of $G$ defined above, we may rewrite (2) in the form

$$
\left\langle\pi_{-s}(x) \mathbf{1}, F(s, \cdot)\right\rangle=\left\langle\pi_{s}(x) 1, F(-s, \cdot)\right\rangle \quad \forall x \in \mathfrak{X} \quad \forall s \in \mathbb{T} .
$$

Let $I_{z}$ denote the normalised intertwining operators between the representations $\pi_{z}$ and $\pi_{-z}$; see [4] or [7]. Then $I_{s} \pi_{s} I_{-s}=\pi_{-s}$, so

$$
\begin{aligned}
\left\langle\pi_{s}(x) 1, F(-s, \cdot)\right\rangle & =\left\langle I_{s} \pi_{s}(x) I_{-s} 1, F(s, \cdot)\right\rangle \\
& =\left\langle\pi_{s}(x) 1, I_{s}^{*} F(s, \cdot)\right\rangle .
\end{aligned}
$$

The set of functions $\left\{\pi_{s}(x) 1: x \in \mathfrak{X}\right\}$ span a dense subspace of $L^{2}(\Omega)$, because $\pi_{s}$ is irreducible, and $I_{s}^{*}=I_{s}^{-1}=I_{-s}$, so we conclude that

$$
F(-s, \omega)=I_{s}^{*} F(s, \omega)=I_{-s} F(s, \omega) .
$$


Next we use the fact that $F(\cdot, \omega)$ is entire of exponential type $N$, and the Paley-Wiener theorem on $\mathbb{Z}$ (which involves contour integration), to write

$$
F(s, \omega)=\sum_{k \in \mathbb{Z}} F(k, \omega) q^{i s k},
$$

where $F(k, \omega)=0$ unless $-N \leq k \leq N$, so that (3) becomes

$$
\sum_{k \in \mathbb{Z}} F(k, \omega) q^{-i k s}=\sum_{k \in \mathbb{Z}}\left(I_{-s} F\right)(k, \omega) q^{i k s} .
$$

Now we apply the difference epcreter $\mathcal{I}_{n}$, defred to be $\mathcal{E}_{n} \quad \mathcal{E}_{n-\mathrm{i}}$ (cos $[7]$, to both sides of this equation: setting $F_{n}(k, \omega)=\mathcal{D}_{n} F(k, \omega)$, so that $F_{n}(k, \omega)=0$ unless $-N \leq k \leq N$, we see that

$$
\sum_{k \in \mathbb{Z}} F_{n}(k, \omega) q^{-i k s}=\sum_{k \in \mathbb{Z}}\left(I_{-s} F\right)_{n}(k, \omega) q^{i k s} .
$$

If $\mathcal{D}_{n} F=F$ then $I_{z} F=c(n, z) F$, where

$$
c(n,-s)= \begin{cases}1 & \text { if } n=0 \\ \frac{1-q^{-1-2 i s}}{1-q^{-1+2 i s}} q^{2 i s n} & \text { if } n \geq 1\end{cases}
$$

(see [7, p. 383]). A straightforward computation shows that

$$
\begin{aligned}
c(n,-s) & =\left(1-q^{-2 i s-1}\right) q^{2 i s n} \sum_{l=0}^{\infty} q^{(2 i s-1) l} \\
& =-q^{2 i s(n-1)-1}+\left(1-q^{-2}\right) \sum_{l=0}^{\infty} q^{2 i s(l+n)-l}
\end{aligned}
$$

when $n \geq 1$. Inserting these expressions for $c(n, z)$ in (4) we obtain, when $n=0$, that $F_{0}(k, \omega)=F_{0}(-k, \omega)$, and when $n \geq 1$,

$$
\sum_{k \in \mathbb{Z}} F_{n}(k, \omega) q^{-i k s}=\sum_{k \in \mathbb{Z}} q^{i k s}\left[q^{2 i s(n-1)-1}+\left(1-q^{-2}\right) \sum_{l=0}^{\infty} q^{2 i s(l+n)-l}\right] F_{n}(k, \omega) .
$$

Taking the Fourier coefficients of both sides, we obtain

$$
F_{0}(k, \omega)=-F_{0}(-k, \omega)
$$


and, when $n \geq 1$,

$$
F_{n}(k, \omega)=-q^{-1} F_{n}(-2 n-k+2, \omega)+\left(1-q^{-2}\right) \sum_{l=0}^{\infty} q^{-l} F_{n}(-2 n-k-2 l, \omega),
$$

for every $k$ in $\mathbb{Z}$ and $\omega$ in $\Omega$.

For fixed $\omega$, we consider the identities (5) and (6) as a system of equations in the unknowns $F_{n}(k, \omega)$. It is easily verified that

(a) if $n>N, F_{n}(k, \omega)=0$ for every $k$ (so that the function $F$ is in fact $N$-cylindrical, and (iv) is a consequence of (i)-(iii));

(b) if $0 \leq n \leq N, F_{n}(k, \omega)=0$ when $k>N+2-2 n$;

(c) for given $n$ and $N$, the functions $F_{n}(k, \omega)$, where $1-n \leq k \leq N+2-2 n$, are determined in terms of the functions $F_{n}(j, \omega)$, where $-N \leq j \leq-n$.

Set $b_{n}=N+1-n$ when $0 \leq n \leq N$. Then, for fixed $n$ and $\omega$, there are at most $b_{n}$ independent $F_{n}(k, \omega)$ 's, and the remaining $F_{n}(k, \omega)$ 's are determined by these.

Now for any given $k$ and $n, \mathcal{D}_{n} F_{n}(k, \omega)=F_{n}(k, \omega)$, so the independent $F_{n}(k, \omega)$ 's can be chosen in at most $d_{n}$ independent ways, where $d_{n}$ is the dimension of the space $\left\{\eta \in C(\Omega): \mathcal{D}_{n} \eta=\eta\right\}$. We therefore conclude that the dimension of the space of functions $F$ satisfying (i)-(iii) is at most

$$
\sum_{n=0}^{N}(N+1-n) d_{n}
$$

But, when $n \geq 1, \mathcal{D}_{n} \eta=\eta$ if and only if $\eta$ is constant on the sets $E(x)$ for every $x$ in $\mathfrak{S}_{n}$ and $\eta$ has zero average on the sets $E(y)$ for every $y$ in $\mathfrak{S}_{n-1}$, while, when $n=0, \mathcal{D}_{0} \eta=\eta$ if and only if $\eta$ is constant on $\Omega$. Thus $d_{n}=e_{n}-e_{n-1}$, where $e_{n}=\left|\mathfrak{S}_{n}\right|$ when $n \geq 0$ and $e_{-1}=0$, and therefore

$$
\sum_{n=0}^{N}(N+1-n) d_{n}=\sum_{k=0}^{N} \sum_{n=0}^{k} d_{n}=\sum_{k=0}^{N} e_{k}=\operatorname{dim} \mathcal{H}\left(C_{N}(\mathfrak{X})\right),
$$

as required.

\section{RAPIDLY DECREASING FUNCTIONS}

We now describe the image of the space $S(\mathfrak{X})$ under $\mathcal{H}$. We say that a function $F: \mathbb{T} \times \Omega \rightarrow \mathbb{C}$ is in the space $C^{\infty}(\mathbb{T} \times \Omega)$ if the function $\partial_{s}^{l} F(s, \omega)$ is in $C(\mathbb{T} \times \Omega)$ for every $l$ in $\mathbb{N}$, and for every $l$ and $k$ in $\mathbb{N}$ there exists a constant $C_{k, l}$ such that

$$
\left\|\partial_{s}^{k}\left(F-\mathcal{E}_{n} F\right)\right\|_{\infty} \leq C_{k, l}(n+1)^{-l} \quad \forall n \in \mathbb{N} \cup\{-1\}
$$

The symbol $C^{\infty}(\mathbb{T} \times \Omega)^{b}$ denotes the subspace of $C^{\infty}(\mathbb{T} \times \Omega)$ of functions which satisfy the symmetry condition (2). 
THEOREM 2. The Helgason-Fourier transformation is an isomorphism from the space $S(\mathfrak{X})$ onto the space $C^{\infty}(\mathbb{T} \times \Omega)^{b}$.

Proof: We show first that if $f$ is in $S(\mathfrak{X})$, then $\tilde{f}$ is in $C^{\infty}(\mathbb{T} \times \Omega)$.

For any $n$ in $\mathbb{N}$, define the averaging operator $\varepsilon_{n}: C(\mathfrak{X}) \rightarrow C(\mathfrak{X})$ by the formula

$$
\varepsilon_{n} f(x)=|\mathfrak{Z}(n, x)|^{-1} \sum_{y \in \mathfrak{Z}(n, x)} f(x) \quad \forall x \in \mathfrak{X}
$$

where

$$
\mathcal{Z}(n, x)= \begin{cases}\{x\} & \text { if }|x| \leq n \\ \{y \in t:|x|=|y|,|c| x, y) \mid \geq n\} & \text { if }|x|>n .\end{cases}
$$

The operators $\varepsilon_{n}$ were introduced in [7], where it was shown that the Poisson transformation intertwines $\mathcal{E}_{n}$ and $\varepsilon_{n}$, that is, for every $\eta$ in $C(\Omega)$ we have

$$
\varepsilon_{n} \mathcal{P}^{z}(\eta)=\mathcal{P}^{z}\left(\mathcal{E}_{n} \eta\right) \quad \forall n \in \mathbb{N} \quad \forall z \in \mathbb{C} .
$$

The identity clearly holds when $\eta$ is replaced by a function $F$ in $C(\mathbb{T} \times \Omega)$, so $\mathcal{H}^{-1}\left(\mathcal{E}_{n} \tilde{f}\right)=\varepsilon_{n} f$ by Fourier inversion, and, equivalently,

$$
\mathcal{E}_{n} \mathcal{H} f=\mathcal{H} \varepsilon_{n} f
$$

Assume now that $f$ is in $S(\mathfrak{X})$ so that, for every $l$ in $\mathbb{N}$, there exists a constant $C_{l}$ such that

$$
|f(x)| \leq C_{l}(|x|+1)^{-l} q^{-|x| / 2} \quad \forall x \in \mathfrak{X} .
$$

Using (7) and the expression of the Poisson kernel in terms of the height function $h_{\omega}$, for all $k$ and $l$ in $\mathbb{N}$ we may write

$$
\begin{aligned}
\partial_{s}^{k}\left(\tilde{f}-\mathcal{E}_{N} \tilde{f}\right)(s, \omega) & =\partial_{s}^{k}\left(\sum_{x \in \mathfrak{X}} q^{(1 / 2+i s) h_{\omega}(x)}\left(f-\varepsilon_{N} f\right)(x)\right) \\
& =\sum_{x \in \mathfrak{X}} i^{k} h_{\omega}(x)^{k} q^{(1 / 2+i s) h_{\omega}(x)}\left(f-\varepsilon_{N} f\right)(x) \\
& =\sum_{x \in \mathfrak{X} \backslash \mathfrak{B}_{N}} i^{k} h_{\omega}(x)^{k} q^{(1 / 2+i s) h_{\omega}(x)}\left(f-\varepsilon_{N} f\right)(x)
\end{aligned}
$$

since $f(x)=\varepsilon_{N} f(x)$ when $x$ is in $\mathfrak{B}_{N}$. Because $h_{\omega}(x) \leq|x|$, and (8) also holds when 
$f$ is replaced by $\varepsilon_{N} f$, we find from (1) that

$$
\begin{aligned}
\left|\partial_{s}^{k}\left(\tilde{f}-\mathcal{E}_{N} \tilde{f}\right)(s, \omega)\right| & \leq \sum_{x \in \mathfrak{X} \backslash \mathfrak{B}_{N}}|x|^{k} q^{h_{\omega}(x) / 2} 2 C_{k+l+3}(|x|+1)^{-k-l-3} q^{-|x| / 2} \\
& \leq 2 C_{k+l+3} \sum_{n=N+1}^{\infty}(n+1)^{-l-3} q^{-n / 2} \sum_{x \in \mathfrak{S}_{n}} q^{h_{\omega}(x) / 2} \\
& \leq 4 C_{k+l+3} \sum_{n=N+1}^{\infty}(n+1)^{-l-2} \\
& \leq 4 C_{k+l+3}(N+1)^{-l}
\end{aligned}
$$

see also [1] where an analogous result for the Radon transformation is proved.

To prove the reverse inclusion, take $F$ in $C^{\infty}(\mathbb{T} \times \Omega)$ which satisfies the symmetry condition (2), so that $F$ is the Helgason-Fourier transform of a function $f$ in $L^{2}(\mathfrak{X})$ by the Plancherel theorem. We shall show that $f$ is in $S(\mathfrak{X})$. Take $x$ in $\mathfrak{X}$, and choose $N$ to be the integer part of $|x| / 3$. Then

$$
|x| / 3<N+1 \leq|x| / 3+1 \text {. }
$$

Write

$$
f=\mathcal{H}^{-1}\left(F-\mathcal{E}_{N} F\right)+\mathcal{H}^{-1}\left(\mathcal{E}_{N} F\right)=\mathcal{H}^{-1} F_{N}+\mathcal{H}^{-1} G_{N}
$$

say. We consider $\mathcal{H}^{-1} F_{N}$ and $\mathcal{H}^{-1} G_{N}$ separately.

First we estimate $\mathcal{H}^{-1} F_{N}$. By assumption, if $k$ is in $\mathbb{N}$, then

$$
\left\|F_{N}\right\|_{\infty} \leq A_{k+1}(N+2)^{-k-1}
$$

so

$$
\begin{aligned}
\left|\mathcal{H}^{-1} F_{N}(x)\right| & \leq \sum_{j=0}^{N} \int_{\mathbb{T}} \int_{E_{j}(x)} q^{j-|x| / 2}\left|F_{N}(s, \omega)\right| d \nu(\omega) d \mu(s) \\
& \leq \sum_{j=0}^{N} q^{j-|x| / 2} \nu\left(E_{j}(x)\right) A_{k+1}(N+2)^{-k-1} \\
& \leq A_{k+1}(N+2)^{-k} q^{-|x| / 2} \\
& \leq 3^{k} A_{k+1}(|x|+1)^{-k} q^{-|x| / 2}
\end{aligned}
$$

from the inversion formula, the normalisation of the Plancherel measure, and (9).

Now we estimate $\mathcal{H}^{-1} G_{N}$. Since $\mathcal{E}_{N}$ commutes with differentiation with respect to $s$ we have

$$
\left|\partial_{s}^{k} G_{N}(s, \omega)\right| \leq B_{k} \quad \forall s \in \mathbb{T} \quad \forall \omega \in \Omega
$$


Recalling that the function $G_{N}(s, \cdot)$ is constant on the sets $E(y)$ for all $y$ in $\mathfrak{S}_{N}$, we denote by $x_{N}$ the point in $\mathfrak{S}_{N}$ on the geodesic path $[o, x]$, by $G_{N}\left(s, x_{N}\right)$ the value that $G_{N}(s, \cdot)$ takes on the set $E\left(x_{N}\right)$, and by $H_{N}(s, \omega)$ the difference $G_{N}(s, \omega)-G_{N}\left(s, x_{N}\right)$. Note that $E\left(x_{N}\right)=\bigcup_{j \geq N} E_{j}(x)$, and that $H_{N}(s, \omega)=0$ when $\omega$ is in $E\left(x_{N}\right)$. Therefore, using the explicit formula for the Poisson kernel, and the integral representation of the spherical functions, we deduce from the inversion formula that

$$
\begin{aligned}
\mathcal{H}^{-1} G_{N}(x)= & \int_{\mathbb{T}} \int_{\Omega} p^{1 / 2-i s}(x, \omega)\left[H_{N}(s, \omega)+G_{N}\left(s, x_{N}\right)\right] d \nu(\omega) d \mu(s) \\
= & \sum_{j=0}^{|x|} \int_{\mathbb{T}} \int_{E_{j}(x)} q^{(1 / 2-i s)(2 j-|x|)} H_{N}(s, \omega) d \nu(\omega) d \mu(s) \\
& \quad+\int_{\mathbb{T}} \int_{\Omega} p^{1 / 2-i s}(x, \omega) G_{N}\left(s, x_{N}\right) d \nu(\omega) d \mu(s) \\
= & \sum_{j=0}^{N-1} \int_{\mathbb{T}} \int_{E_{j}(x)} q^{(1 / 2-i s)(2 j-|x|)} H_{N}(s, \omega) d \nu(\omega) d \mu(s) \\
& \quad+\int_{\mathbb{T}} \phi_{-s}(x) G_{N}\left(s, x_{N}\right) d \mu(s) \\
= & \sum_{j=0}^{N} I_{j, N}(x) \quad \forall x \in \mathfrak{X} \backslash \mathfrak{B}_{N-1}
\end{aligned}
$$

where

$$
I_{j, N}(x)=\int_{\mathbb{T}} \int_{E_{j}(x)} q^{(1 / 2-i s)(2 j-|x|)} H_{N}(s, \omega) d \nu(\omega) d \mu(s)
$$

if $0 \leq j \leq N-1$ and

$$
I_{N, N}(x)=\int_{\mathbb{T}} \phi_{-s}(x) G_{N}\left(s, x_{N}\right) d \mu(s) .
$$

We claim that for every $l$ in $\mathbb{N}$ there exists a constant $C_{l}$, which depends on $l, q$ and $B_{k}$ (where $0 \leq k \leq l+1$ ), but not on $f, x$, or $N$, such that

$$
\left|I_{j, N}(x)\right| \leq C_{l}(|x|+1)^{-l-1} q^{-|x| / 2} \quad \forall j \in\{0,1, \ldots, N\} .
$$

Assuming our claim, the estimate required to finish the proof of the theorem follows immediately: indeed, from (8) we conclude that

$$
\left|\mathcal{H}^{-1} G_{N}(x)\right| \leq(N+1) C_{l}(|x|+1)^{-l-1} q^{-|x| / 2} \leq C_{l}(|x|+1)^{-l} q^{-|x| / 2} .
$$

To finish, we must prove our claim. We estimate $I_{j, N}$ where $0 \leq j \leq N-1$. To deal with $I_{N, N}$ one argues similarly, using the explicit expression of the spherical 
functions $\phi_{s}$. Recalling that $d \mu(s)=c_{G}|\mathbf{c}(s)|^{-2} d s$, and noting that all the functions involved are smooth in $s$, we integrate by parts and find that $I_{j, N}$ is equal to

$$
c_{G} \frac{q^{j-|x| / 2} i^{l+1}}{(2 j-|x|)^{l+1} \log ^{l+1} q} \int_{\mathbb{T}} q^{-i s(2 j-|x|)} \partial_{s}^{l+1}\left(|\mathbf{c}(s)|^{-2} \int_{E_{j}(x)} H_{N}(s, \omega) d \nu(\omega)\right) d s .
$$

By Leibniz's rule, this is a linear combination with coefficients $c_{G}\left(\begin{array}{c}l+1 \\ k\end{array}\right)$ of $l+2$ terms of the form

$$
\frac{q^{j-|x| / 2} i^{l+1}}{(2 j-|x|)^{l+1} \log ^{l+1} q} \int_{\mathbb{T}} q^{-i s(2 j-|x|)} \partial_{s}^{l+1-k}\left(|\mathbf{c}(s)|^{-2}\right) \int_{E_{j}(x)} \partial_{s}^{k} H_{N}(s, \omega) d \nu(\omega) d s .
$$

Using the estimate $\nu\left(E_{j}(x)\right) \leq q^{-j}$, it is easily shown that the absolute value of each term is bounded above by

$$
\begin{gathered}
\frac{q^{j-|x| / 2}}{(|x|-2 j)^{l+1} \log ^{l+1} q} \int_{\mathbb{T}}\left|\partial_{s}^{l+1-k}\left(|\mathbf{c}(s)|^{-2}\right)\right| 2 B_{k} \nu\left(E_{j}(x)\right) d s \\
\leq \frac{2 B_{k} 3^{l+1 q^{-|x| / 2}}}{|x|^{l+1} \log ^{l+1} q} \int_{\mathbb{T}}\left|\partial_{s}^{l+1-k}\left(|\mathbf{c}(s)|^{-2}\right)\right| d s,
\end{gathered}
$$

and the required estimate for $I_{j, N}$ follows.

\section{REFERENCES}

[1] W. Betori, J. Faraut and M. Pagliacci, 'An inversion formula for the Radon transform on trees', Math. Zeit. 201 (1989), 327-337.

[2] E. Casadio Tarabusi, J.M. Cohen and F. Colonna, 'The range of the horocyclical Radon transform on homogeneous trees', preprint.

[3] M. Cowling, S. Meda and A. G. Setti, 'An overview of harmonic analysis on the group of isometries of a homogeneous tree', Exposit. Math. 16 (1998), 385-424.

[4] A. Figà-Talamanca and C. Nebbia, Harmonic analysis and representation theory for groups acting on homogeneous trees, London Math. Soc. Lecture Notes Series, 162 (Cambridge Univ. Press, Cambridge, 1991).

[5] A. Figà-Talamanca and M. Picardello, Harmonic analysis on free groups (Dekker, New York, 1983).

[6] S. Helgason, Geometric analysis on symmetric spaces, Math. Surveys and Monog. (Amer. Math. Soc., Providence, R.I., 1994).

[7] A. M. Mantero and A. Zappa, 'The Poisson transform and representations of a free group', J. Funct. Anal. 51 (1983), 372-399.

School of Mathematics

University of New South Wales

Sydney NSW 2052

Australia

e-mail: m.cowling@unsw.edu.au

\author{
Università dell'Insubria-Polo di Como \\ Facoltà di Scienze \\ via Lucini 3 \\ I-22100 Como \\ Italy \\ e-mail: setti@fis.unico.it
}

